\title{
Search for a diffuse cosmic neutrino flux with ANTARES using track and cascade events
}

\author{
Jutta Schnabel on behalf of the ANTARES collaboration \\ Erlangen Centre for Astroparticle Physics, Erwin-Rommel Str. 1, 91052 Erlangen, Germany \\ E-mail: jutta.schnabel@fau.de
}

\section{Steffen Hallmann*}

Erlangen Centre for Astroparticle Physics, Erwin-Rommel Str. 1, 91052 Erlangen, Germany

E-mail: steffen.hallmannefau.de

\begin{abstract}
The ANTARES neutrino telescope has since its final deployment in 2008 contributed to the searches for high-energy neutrino sources. In this work, prior ANTARES searches for the diffuse events from track-like charged-current muon neutrinos as well as cascade-like interaction from all neutrino flavours are integrated into a new comprehensive all-flavour search. The method employs a multivariate analysis approach on six years of ANTARES data optimizing for the discovery of a cosmic neutrino flux as observed by the IceCube experiment. This analysis reaches at its first stage a sensitivity of $\Phi_{I C 2.5} E^{2.5}=5.4 \times 10^{-6} \mathrm{GeV} \mathrm{cm}^{-2} \mathrm{sr}^{-1} \mathrm{~s}^{-1}$ and observes a slight excess of events over the background estimation.
\end{abstract}

The 34th International Cosmic Ray Conference,

30 July- 6 August, 2015

The Hague, The Netherlands

${ }^{*}$ Speaker. 


\section{Introduction}

The search for neutrinos of cosmic origin has evolved greatly in the last few years. As decay products of, among others, $\pi$ and $K$ mesons, neutrino production is expected to occur in astrophysical sources through interaction of hadrons. At cosmic acceleration sites, the interaction of protons accelerated through shock acceleration are expected to lead to a cosmic neutrino flux that follows the distribution of the cosmic ray spectrum [1]. As hadrons from cosmic rays also lead to air showers in Earth's atmosphere, this cosmic neutrino component needs to be distinguished from an atmospheric background of neutrinos from both conventional atmospheric neutrinos [2] and especially high-energy neutrinos emitted from prompt decays of hadrons containing charm quarks in the atmosphere [3].

ANTARES has already set a limit on this diffuse flux of cosmic neutrinos from charged-current interactions of $v_{\mu}$ [4]. An excess of diffuse cosmic neutrinos was recently measured for all neutrino flavours by the IceCube experiment [5]. After several more years of data taking and further development of reconstruction techniques for events from all neutrino flavours, the ANTARES sensitivity towards the cosmic neutrino flux has increased significantly, although the approach to the measurement of the cosmic neutrino flux must differ to that of IceCube due to the different technical conditions. In this work the first combined search for neutrinos of all flavours is presented by applying a new methodology which focuses on multivariate techniques in order to incorporate the different event topologies.

\section{Neutrino measurement with ANTARES}

At the ANTARES [8] site at about $2.5 \mathrm{~km}$ below sea level off the French Mediterranean coast, the measurement of neutrinos is challenged by two main factors. On the one hand, the 12 detection lines are not only subject to the sea current and varying environmental conditions, but also detect photons from ambient light emitters like ${ }^{40} K$ decays and, to a larger extent, bioluminescent sea life. To handle this, effective event selection and triggering schemes are in place, of which only the more stringent ones are used in this analysis to ensure a low influence of sea conditions on the event selection. On the other hand, muons produced in atmospheric air showers penetrate the overburden of water such that at the detector level they outnumber neutrino-induced events by about $1: 10^{6}$.

\subsection{Event Simulation and Data Selection}

Due to the varying environmental conditions, event simulation in ANTARES [6], [7] is done on a run-by-run basis, accounting for changing bioluminescence rates within run periods of a few hours. Due to the complex environmental conditions, the agreement between data and simulation naturally varies, which is accounted for in the analysis procedure by restricting the optimization on simulation to runs which show a good agreement between data and simulation for all relevant parameter distributions. Consequently, an effective livetime of 913 days is selected from the data taking period between 2007 and 2013. A large amount of the remaining data in the same period has also good quality, but lacks an according run by run simulation. The total amount of available data is 1700 days including the previously described selection, for which the analysis was optimized. 


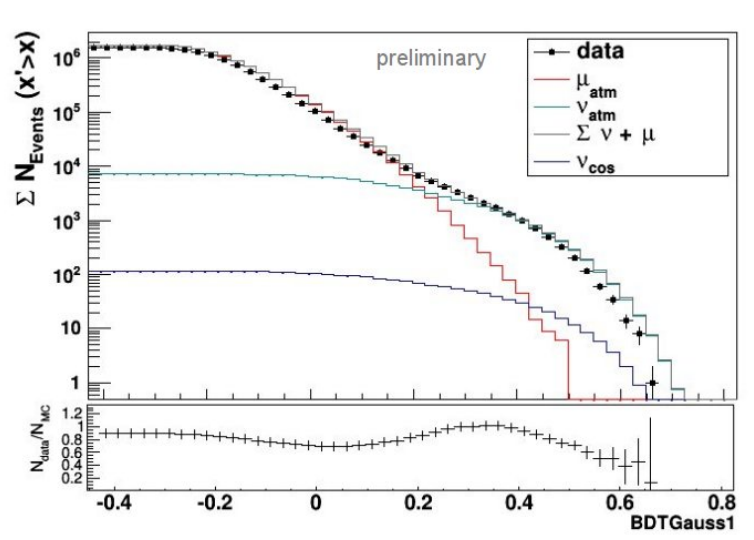

(a) BDT for $\mu_{\text {atm }}$ suppression

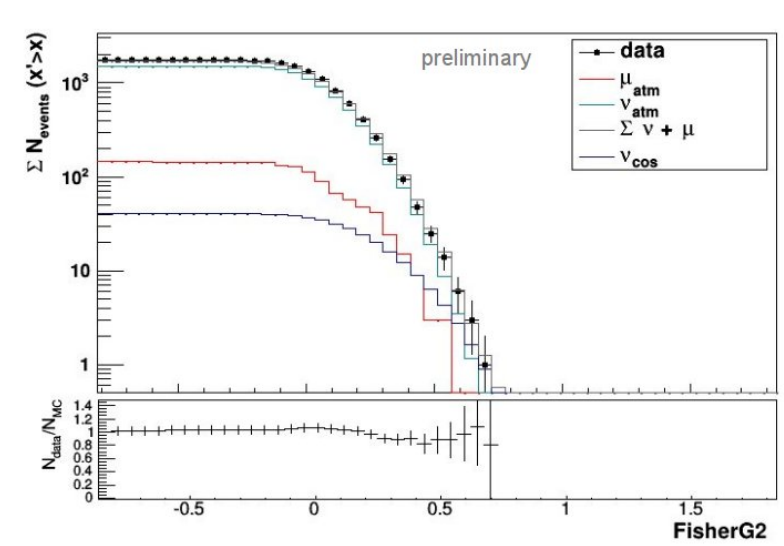

(b) Fisher discriminant for $v_{c o s}$ identification

Figure 1: Agreement between data and simulation for 913 days for a) the BDT method for atmospheric muon suppression and b) the Fisher discriminant for cosmic neutrino identification after a cut on $B D T>0.345$

The remaining part will be included in a consecutive step which is still in progress at the time of this presentation.

\subsection{Event identification and reconstruction}

Event topologies seen in the ANTARES detector are divided into charged-current $v_{\mu}$ interactions which mainly produce Cherenkov emission along the extensive muon track, and cascades of short-lived secondary particles producing photon emission at the interaction point of $v_{e}$ and neutral-current $v_{\mu}$. For these track-like and cascade-like events specialized event reconstruction methods have been developed, including likelihood-based directional reconstruction from photon hit patterns and various track energy estimators. Although no special reconstruction of $v_{\tau}$ events was used in this work, their topology varies between cascade-like events for neutral current interactions and short track-like events for charged-current interactions producing a quickly decaying $\tau$ lepton resulting in a track-like $\mu$ or cascade, making it possible to reconstruct $\nu_{\tau}$ events with existing track and cascade reconstruction techniques.

In order to incorporate all event signatures in a search for a diffuse cosmic neutrino flux, multivariate techniques [9] were employed to identify the relevant features from both track-like and cascade-like events. As the search for cosmic neutrino events in ANTARES can roughly be divided into firstly distinguishing the atmospheric muon events from neutrino-induced events and secondly extracting the cosmic signal from the atmospheric neutrino background, two multivariate tools were used to fulfil these tasks.

\subsection{Atmospheric muon suppression}

The distinction between atmospheric muons entering the detector from above and high-energy neutrino-induced events coming from all directions can most effectively be accomplished by a combination of event angular estimates and the quality of cascade and track reconstruction methods with energy-related variables. 


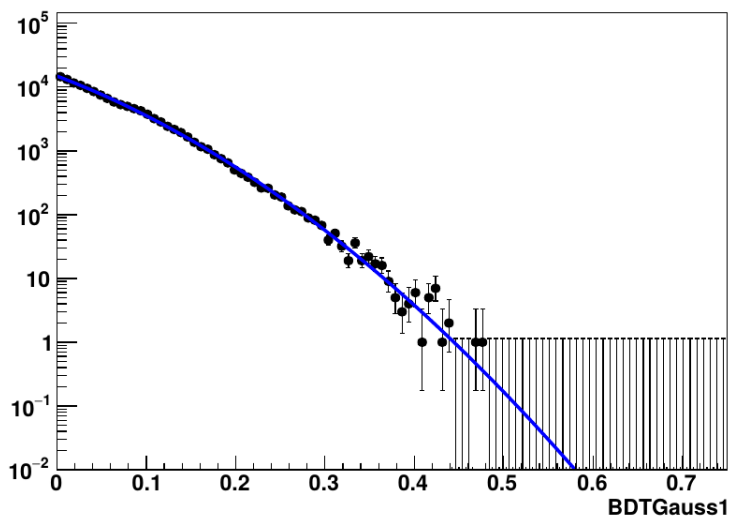

(a) Muon fit for BDT

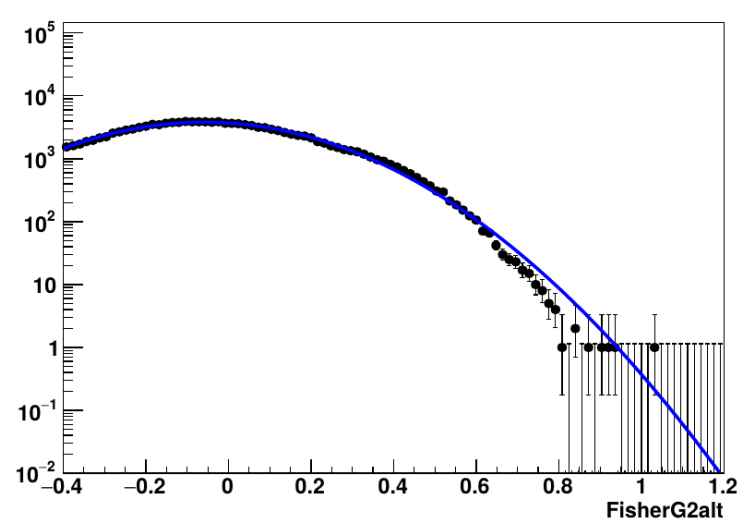

(b) Muon fit for Fisher discriminant

Figure 2: Projections of the atmospheric muon distribution and the according extrapolation for the BDT and Fisher discriminant.

In order to find the most effective parameter combination for this task, candidate parameters and multivariate methods were tested in an optimization process employing the signal-background separation $S=\left(\mu\left(x_{s i g}\right)-\mu\left(x_{b k g}\right)\right) /\left(R M S\left(x_{s i g}\right)-R M S\left(x_{b k g}\right)\right)$ as optimization parameter, with $\mu$ denoting the mean and $R M S$ the root mean square of the parameter $x$ in signal and background events. Of several multivariate methods, Boosted Decision Trees (BDT) [9] ranked among the best performing. Following a parameter scanning procedure, nine parameters were selected as input parameters. These included two track zenith angle estimates, one track and one cascade reconstruction quality parameter, a track energy estimate and the number of photon hits measured in all PMTs in a cascade, one atmospheric muon suppression parameter and two geometrical parameters describing the extension of the event within the detector and the time residual distribution of the photons. The behaviour of the resulting BDT can be seen in Figure 1a, where the excess of atmospheric neutrinos, weighted according to the Honda [2] atmospheric neutrino flux model over the background of atmospheric muons can be seen at high BDT values.

\subsection{Cosmic neutrino identification}

The distinction between atmospheric and cosmic neutrino events is to the largest extent achieved through determining the neutrino energy, as the cosmic neutrino flux is expected to follow a harder spectrum than the background of atmospheric events. The additional energy deposited in the detector from neutrino interactions is seen as additional light yield originating from photons from either the Cherenkov emission from secondary particles at the interaction vertex or as radiation from energy loss processes along the muon track. Therefore, the number of photons, measured as charge collected on the photomultipliers, gives the simplest representation of the energy information.

As various sophisticated energy estimators were developed within ANTARES for the different event types, another multivariate technique was employed to arrive at a common estimate for the signal-likeness of any neutrino event. Here, the signal efficiency $\varepsilon$ at very small background was employed as optimization parameter in the search for best parameters and multivariate methods, as the task of signal extraction demands a high purity of the final event sample. The following testing 

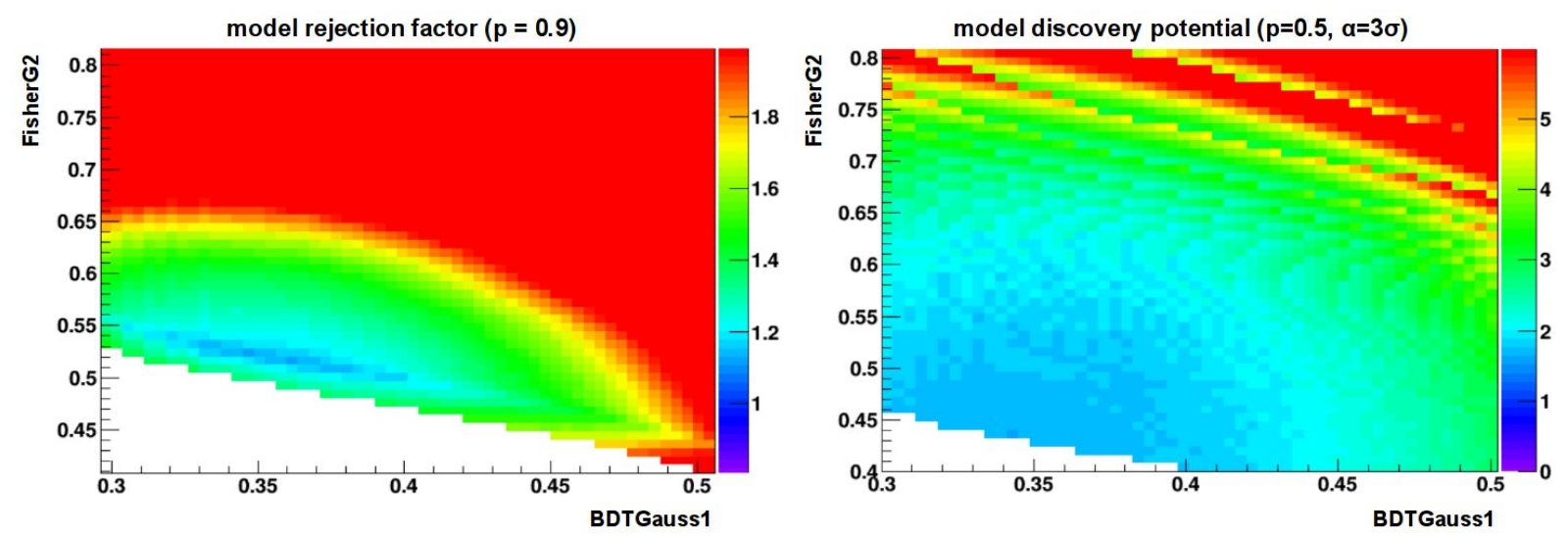

Figure 3: Model rejection factor (left) and discovery potential (right) (for $3 \sigma$ at 50\%) for various event cut configurations, employing both TMVA methods with a Gaussian preprocessing [9]. The compromise discussed below was set for the best MRF at BDT $>0.345$ and Fisher $>0.52$

showed simple linear estimators to perform well for this task, leading to the use of a Fisher discriminant [9], which combined three different energy estimates for tracks and cascades, three photon counts from different event-type specific photon hit selections, as well as a cascade zenith angle estimate, one track and one cascade reconstruction quality parameter and the number of storeys used for the cascade reconstruction, which adds geometrical information to the estimator. The behaviour of the Fisher discriminant can be seen in Figure 1b, using a prior cut on the BDT parameter to reduce the contamination of the event sample by atmospheric muons to $\approx 10 \%$.

\section{Analysis procedure}

Having obtained tools for the suppression of both the atmospheric muon and atmospheric neutrino background, the analysis procedure can be reduced to a simple search for the optimal combination of parameter cuts on these two multivariate parameters. As the sensitivity of ANTARES is, by extrapolation from previous results, expected to come close to the flux of cosmic neutrinos observed by IceCube, the selection of the optimal cuts should both fulfil the requirements of a model discovery and a model rejection technique [10].

\subsection{Signal optimization}

In order to perform the signal optimization as accurately as possible, a fit on the distribution of the atmospheric muon component was introduced as well as a prompt atmospheric neutrino flux following [3]. The fit is necessary due to the limited statistics of the simulated atmospheric muon sample, which only accounts for $1 / 3$ of the total data taking time. Here, a two-dimensional Gaussian function was fit to the atmospheric muon distribution for both multivariate parameters (blue lines in Figure 2), introducing the uncertainty of the fit parameter propagated to the muon number as error on the estimated atmospheric muon number. The contribution of $v_{\tau}$ events was estimated from a small simulation and not included in the optimization procedure. The procedure was therefore performed for $v_{e}$ and $v_{\mu}$ events from a cosmic signal according to the IceCube measurement [5], 


\begin{tabular}{|l|r|r||l|r|r|}
\hline Signal & $N_{\text {events }}$ & error & Background & $N_{\text {events }}$ & error \\
\hline$N^{2.5} v_{\mu, C C}$ & 1.4 & $0.37^{b}+0.57^{c}$ & $N_{\text {Honda }} v_{\mu, C C}$ & 5.3 & $2.29^{b}$ \\
$N^{2.5} v_{\mu, N C}, v_{e}$ & 2.6 & $0.63^{b}+0.06^{c}$ & $N_{\text {Honda }} v_{\mu, N C}, v_{e}$ & 2.4 & $0.9^{b}+0.1^{c}$ \\
$N^{2.5} v_{\tau}$ & 0.9 & $0.53^{d}$ & $N_{\text {Enberg }} v_{\mu, C C}$ & 0.2 & $0.08^{b}+0.45^{c}$ \\
$N^{2.0} v_{\mu, C C}$ & 1.8 & & $N_{\text {Enberg }} v_{\mu, N C}, v_{e}$ & 0.6 & $0.14^{b}+0.01^{c}$ \\
$N^{2.0} v_{\mu, N C}, v_{e}$ & 2.2 & & $N_{\text {Enberg }} v_{\tau}$ & 0.01 & 0.0 \\
$N^{2.0} v_{\tau}$ & 0.6 & & $N \mu_{\text {atm }}$ & 1.0 & $0.15^{a}$ \\
\hline$\Sigma$ & $5.0^{(2.5)} / 4.5^{(2.0)}$ & \pm 1.1 & $\Sigma$ & 9.5 & \pm 2.5 \\
\hline
\end{tabular}

Table 1: Signal and background expectation including error estimates for 913 days of ANTARES lifetime. As cosmic flux, the IceCube measurement [5] is used assuming either a spectral index $\lambda=$ 2.5 or $\lambda=2.0$. Error estimates are drawn from a) error on muon fit parameters, b) water absorption length uncertainty, c) water scattering length uncertainty, d) difference between $\tau$ estimate and toy simulation.

assuming $\Phi_{I C 2.5} E^{2.5}=4.1 \times 10^{-6} \mathrm{GeV} \mathrm{cm}^{-2} \mathrm{sr}^{-1} \mathrm{~s}^{-1}$, with atmospheric neutrinos simulated using the conventional flux from [2] and including the extrapolated muon number. Intending to ultimately use this analysis on the full data sample of 1700 days, event numbers were scaled to this livetime for the event selection optimization. As can be seen in Figure 3, a model rejection optimization then leads to an optimal result that still exhibits a good model discovery potential, as both minimal regions overlap.

\subsection{Error estimates}

In order to account for simulation uncertainties in the standard ANTARES simulation, the uncertainty of water propagation properties, i.e. the water absorption and scattering length, was estimated on a simulation including 12 days data taking and the difference in event numbers after final cuts from variation of these properties by $10 \%$ is taken into account.

Also, a small simulation of $v_{\tau}$ events equivalent to 12 days was produced to estimate the behaviour of these events in the analysis. As the event topology does to a large extent agree with that of cascade events, the $v_{\tau}$ contribution could also be extrapolated from cascade simulations as done in [11]. As both methods have limited accuracy, the $v_{\tau}$ simulation was used to estimate this contribution, while the difference between event numbers from both methods was introduced as error. The errors drawn from these estimates are, together with the final event numbers, shown in Table 1.

\section{Results}

The search for a diffuse flux of cosmic neutrinos in ANTARES leads to the expected event numbers given in Table 1 over the background of conventional [2] and prompt [3] neutrino flux. Assuming a spectral index of -2.5 and the cosmic neutrino flux per flavour as $\Phi_{I C 2.5} E^{2.5}=4.1 \times$ $10^{-6} \mathrm{GeV} \mathrm{cm}^{-2} \mathrm{sr}^{-1} \mathrm{~s}^{-1}$, a sensitivity of $\Phi_{90 \% I C 2.5}=1.33 \Phi_{I C 2.5}$ between $6.8 \mathrm{TeV}$ and $1.1 \mathrm{PeV}$ is reached for 913 days. Accordingly, a harder spectrum of $\Phi_{I C 2.0} E^{2.0}=1.1 \times 10^{-8} \mathrm{GeV} \mathrm{cm}^{-2} \mathrm{sr}^{-1} \mathrm{~s}^{-1}$ following the spectral index of [1] and the magnitude of [5] reaches a sensitivity per flavour of $\Phi_{90 \% I C 2.0}=1.6 \times 10^{-8} \mathrm{GeV} \mathrm{cm}^{-2} \mathrm{sr}^{-1} \mathrm{~s}^{-1}$, valid within $18 \mathrm{TeV}$ to $7.5 \mathrm{PeV}$. 


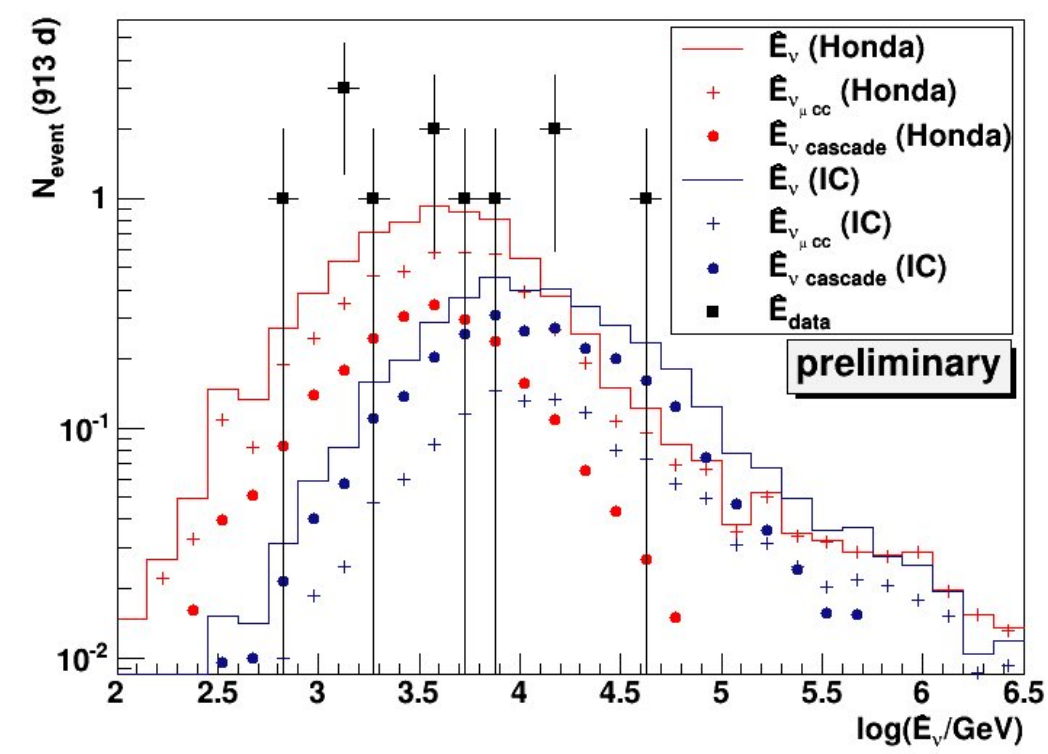

Figure 4: Energy distribution for the events found in 913 days, giving the reconstructed vertex energy $\hat{E}$ by a cascade reconstruction [11] for data and simulated $v_{e}$ and $v_{\mu}$ contributions

In 913 days of ANTARES data, 12 events were found, which is a slight excess over the background expectation of 9.5 events. The events studied in this analysis generally exhibit similar event topology which allows each to be reconstructed as both track and cascade events. As the events are found to be either interacting close to the detector or inside the instrumented volume, the number of photons measured by the detector is generally large. However, the various energy reconstruction methods vary in the interpretation of the neutrino energy depending on their event signature assumption, as e.g. track energy reconstructions generally interpret the energy deposition as one of several catastrophic energy losses and therefore assign a higher primary neutrino energy. In Figure 4, the cascade vertex energy is shown for the final events. Including error estimates according to [13], upper limits on the respective fluxes can be set as $\Phi_{90 \% \text { u.l.IC2.5 }}=2.4 \Phi_{I C 2.5}$ and $\Phi_{90 \% \text { u.l.IC2.0 }}=2.6 \Phi_{I C 2.0}$. These results are compared to previous analyses and the flux measured by IceCube [5] in Figure 5.

This first analysis step shows the capability of ANTARES to combine the former separate searches for a diffuse cosmic neutrino flux through multivariate methods into an effective analysis of all neutrino event types. As the analysis presented here only incorporates a little more than half of the data taken by the ANTARES experiment until end 2013, a full analysis can be expected to reach a sensitivity similar to the flux measured by IceCube.

\section{References}

[1] E. Waxman and J. Bahcall, High energy neutrinos from astrophysical sources: An upper bound, Phys. Rev. D 59, 023002 (1998)

[2] M. Honda et al., Calculation of atmospheric neutrino flux using the interaction model calibrated with atmospheric muon data, Phys. Rev. D 75, 043006 (2007)

[3] R. Enberg et al., Prompt neutrino fluxes from atmospheric charm, Phys. Rev. D 78, 043005 (2008) 


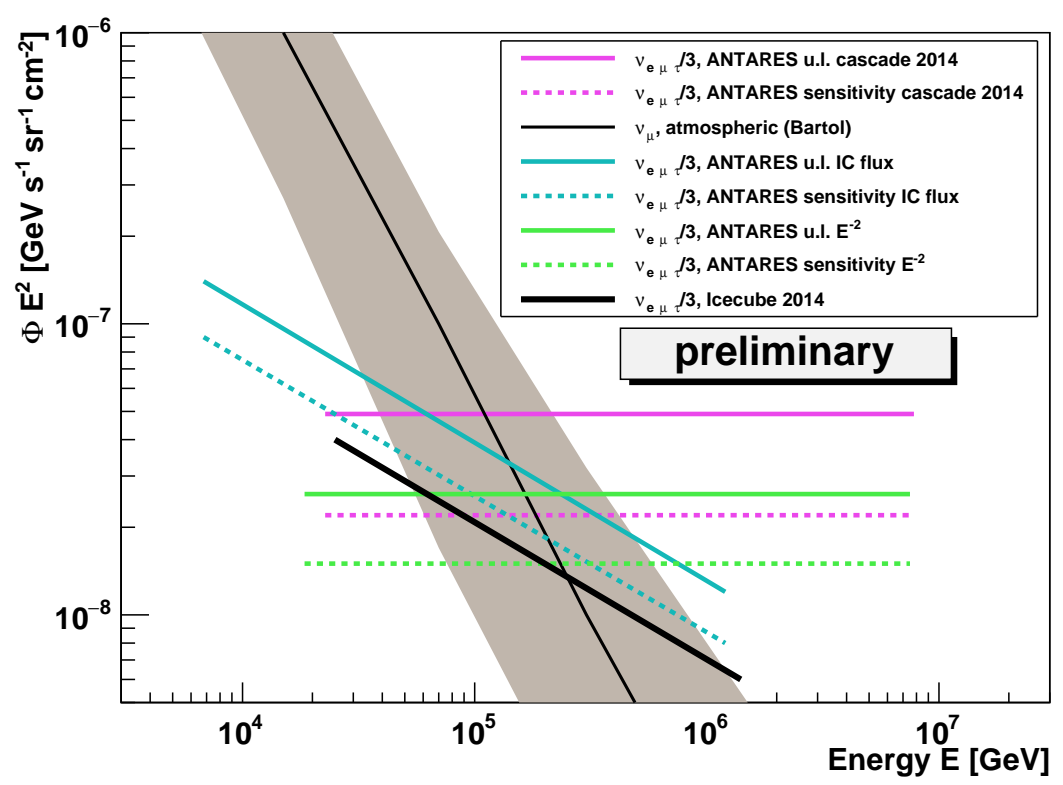

Figure 5: Flux limits for the last track and cascade analyses in ANTARES, the Icecube result and limits and sensitivity of this most recent analysis

[4] J.A. Aguilar et al., Search for a diffuse flux of high-energy $v_{\mu}$ with the ANTARES neutrino telescope, Phys. Letters B, Vol. 696, (2011)

[5] M. G. Aartsen et al., Atmospheric and Astrophysical Neutrinos above 1 TeV Interacting in IceCube, Phys. Rev. D 91, 022001 (2015)

[6] G. Carminati et al., MUPAGE: a fast atmospheric MUon GEnerator for neutrino telescopes based on PArametric formulas, arXiv:0907.5563 [astro-ph.IM] (2009)

[7] J. Brunner, ANTARES simulation tools, Proceedings of the First VLVnT Workshop, Amsterdam (2003)

[8] M. Ageron et al., ANTARES : The first undersea neutrino telescope, Nucl. Instrum. Meth. A 656 11-38, arXiv:1104.1607 (2011)

[9] A. Hoecker et al., TMVA - Toolkit for Multivariate Data Analysis, PoS ACAT 040 (2007), arXiv:physics/0703039

[10] G. C. Hill et al., Examining the balance between optimizing an analysis for best limit setting and best discovery potential, PHYSTAT05, DOI: 10.1142/9781860948985_0025 (2006)

[11] F. Folger, Search for a diffuse cosmic neutrino flux using shower events in the ANTARES neutrino telescope, $\mathrm{PhD}$ thesis, FAU Erlangen (2014)

[12] A. Kouchner, Recent results from the ANTARES neutrino telescope, ICRC 2013, arXiv:1312.4308 [astro-ph.HE] [2013]

[13] J. Conrad, A program for confidence interval calculations for a Poisson process with background including systematic uncertainties: POLE 1.0, Computer Physics Communications 158, p. 117-123 (2004) 